\title{
Liver Stiffness Measurement Is Useful to Predict Early Recurrence of Hepatocellular Carcinoma after Sustained Virological Responses by Direct Antiviral Agents in Patients with Hepatitis C
}

\author{
Noboru Hirashima ${ }^{*}$, Hiroaki Iwase1, Masaaki Shimada1, Noboru Urata1, \\ Etsuko Iio², Yasuhito Tanaka² \\ ${ }^{1}$ Department of Gastroenterology, National Hospital Organization, Nagoya Medical Center, Nagoya, Japan \\ ${ }^{2}$ Department of Virology and Liver Unit, Nagoya City University, Graduate School of Medical Sciences, Nagoya, Japan \\ Email: ‘hirasima@nnh.hosp.go.jp, iwaseh@nnh.hosp.go.jp, shimada@nnh.hosp.go.jp,n_urata@nnh.hosp.go.jp, \\ eiio@med.nagoya-cu.ac.jp, ytanaka@med.nagoya-cu.ac.jp
}

How to cite this paper: Hirashima, N., Iwase, H., Shimada, M., Urata, N., Iio, E. and Tanaka, Y. (2018) Liver Stiffness Measurement Is Useful to Predict Early Recurrence of Hepatocellular Carcinoma after Sustained Virological Responses by Direct Antiviral Agents in Patients with Hepatitis C. Open Journal of Gastroenterology, 8, 255-268.

https://doi.org/10.4236/ojgas.2018.87028

Received: June 1, 2018

Accepted: July 24, 2018

Published: July 27, 2018

Copyright $\odot 2018$ by authors and Scientific Research Publishing Inc. This work is licensed under the Creative Commons Attribution International License (CC BY 4.0).

http://creativecommons.org/licenses/by/4.0/

\section{(c) (i) Open Access}

\begin{abstract}
Background: Recently, increases in the risk of Hepatocellular carcinoma (HCC) recurrence in patients with hepatitis $\mathrm{C}$ virus (HCV) due to the administration of direct antivirals agents (DAA) have been reported. Methods: One hundred and nineteen patients who were treated with DAA and achieved sustained viral response (SVR) were prospectively followed-up for over two years by transient elastography with liver stiffness measurements (LSM). Fourteen out of 119 patients (12\%) had a history of being treated for HCC by radiofrequency ablation or resection and achieved complete responses after previous HCC treatments before the initiation of DAA. HCC was diagnosed by contrast-enhanced computed tomography $(\mathrm{CT})$ or enhanced magnetic resonance imaging (MR). CT or MR was performed before the DAA treatment and every 6 months after during the follow-up. LSM was performed at the initiation of DAA (LSM0), at 24 weeks after the start of DAA (LSM24), at 48 weeks after that (LSM48) and at 2 years after that (LSM2y). Results: LSM0, LSM24, LSM48 and LSM2y of 105 patients without HCC were 7.5 (3.0 - 27.0), 6.0 (2.5 - 31.6), $4.6(2.6-25.2)$ and $4.4(3.1-29.9) \mathrm{kPa}$, respectively, showing significant improvements. Three out of 105 patients (2.9\%) subsequently developed HCC and their LSM showed improvements. Eight out of fourteen patients (57\%) with a history of HCC treatments subsequently developed HCC recurrence. LSM0 in the eight patients with recurrence increasing from 12.1 to $27.0 \mathrm{kPa}$,
\end{abstract}


LSM24 from 9.9 to $26.6 \mathrm{kPa}$ and LSM48 from 9.6 to $18.0 \mathrm{kPa}$. On the other hand, the six other patients without recurrence had LSM values that were less than $12.0 \mathrm{kPa}$ at last. Based on the ROC analysis, LSM0 15.4, LSM24 12.8 and LSM48 $9.6 \mathrm{kPa}$ were identified as cut-off values. Conclusion: HCV patients previously treated for HCC with high LSM values before and after DAA have an elevated risk of HCC recurrence, particularly LSM24 > $12.8 \mathrm{kPa}$ and LSM48 > $9.6 \mathrm{kPa}$.

\section{Keywords}

Hepatitis C Virus Infection, Direct-Acting Antivirals, Hepatocellular Carcinoma, Recurrence, Liver Stiffness Measurement

\section{Introduction}

Recently, interferon (IFN)-free direct-acting antiviral agents (DAA) have improved the rates of sustained virological responses (SVR) in the HCV-infected patients [1]-[6]. In Japan, daclatasvir (DCV) plus asunaprevir (ASV) was initially approved for chronic hepatitis C virus (HCV) genotype 1 patients in September 2014 [5], followed by sofosbuvir (SOF) plus ledipasvir (LDV) in September 2015 [4] and ombitasvir (OBV) plus paritaprevir (PTV) with ritonavir (rit) in November 2015 [7]. Furthermore, SOF plus ribavirin (RBV) was approved for chronic HCV genotype 2 patients in May 2015 [8]. Without a previous history of simeprevir therapy and pre-existing Non-structural protein 5A (NS5A) $\mathrm{Y} 93 \mathrm{H}$, DCV plus ASV was reported to be about $90 \%$ effective for patients with $\mathrm{HCV}$ genotype $1 b$ [9]. DAA were recently shown to achieve SVR in more than $90 \%$ of $\mathrm{HCV}$-infected patients even in liver cirrhosis patients with HCV. However, DAA has been reported to increase the risk of hepatocellular carcinoma (HCC) in $\mathrm{HCV}$-infected patients, particularly in those received DAA after the treatment of HCC, potentially causing "HCC recurrence" [10]. SVR decreased the long-term risk of HCC in patients with advanced fibrosis in previous studies on IFN-based treatments for chronic hepatitis C [11]. On the other hand, liver stiffness measurements (LSM) have been significantly reduced by SVR following both IFN-based therapy [12] and DAA therapy [13] [14]. In the DAA therapy HCC recurrence more significantly recognized in the cirrhotic patients with LSM values over $21.5 \mathrm{kPa}$ before DAA therapy [15]. We reported that chronic hepatitis C patients previously treated for HCC with high LSM values before and after DCV plus ASV might be at elevated risk of HCC recurrence [16]. DCV plus ASV was approved only in Japan and Korea of over the world; therefore the aim of the present study was to prospectively evaluate the relationship between the recurrence of HCC and liver stiffness values by transient elastography after the successful treatment of patients with $\mathrm{HCV}$ infection with all DAA containing SOF plus LDV, OBV plus PTV with rit and SOF plus RBV. 


\section{Methods}

This study included a total of 119 HCV-infected patients treated with DAA (DCV plus ASV 43, SOF plus LDV 39, SOF plus RBV 34, OBV plus PTV with rit 3) between November 2014 and June 2016 and achieving SVR were prospectively followed-up for over two years. Fourteen out of 119 patients (12\%) had a history of being treated for HCC by radiofrequency ablation (RFA) or resection and achieved complete responses after previous HCC treatments before the initiation of DAA. DCV at $60 \mathrm{mg}$ and ASV at $100 \mathrm{mg}$ were administered daily to patients infected with genotype $1 \mathrm{~b}$ for 24 weeks between December 2014 and April 2016. NS5A variants were confirmed to be absent in all patients before the initiation of DCV plus ASV because the variants associated with Y93 and L31 in the NS5A region are known to be the most strongly associated with treatment outcomes [5] [17] [18]. Forty-three out of 45 patients (96\%) treated with DCV plus ASV achieved SVR. SOF at $400 \mathrm{mg}$ plus LDV at $90 \mathrm{mg}$ were administered daily to patients infected with genotype 1 for 12 weeks between September 2015 and June 2016. All 39 patients (100\%) treated with SOF plus LDV achieved SVR. SOF at $400 \mathrm{mg}$ plus RBV at $200-600 \mathrm{mg}$ were administered daily to patients infected with genotype 2 for 12 weeks between September 2015 and June 2016. Thirty-four out of 36 patients (94\%) treated with SOF plus RBV achieved SVR. OBV at $25 \mathrm{mg}$ plus PTV at $150 \mathrm{mg}$ with rit at $100 \mathrm{mg}$ were administered daily to patients infected with genotype 1 for 12 weeks between April 2016 and June 2016, and 3 out of 3 patients (100\%) achieved SVR.

The baseline characteristics of the patients included in this study are shown in Table 1. Fib-4 score was as follows: (Age $\times$ AST) $/\left(\right.$ Platelets $\times 10 \times$ ALT $^{1 / 2}$ ) Table 2 shows the laboratory data of 14 cases with previous HCC histories before and after the DAA treatment. Blood chemistry examinations and patient interviews were conducted every two weeks after the initiation of the treatment to detect adverse events. Virological responses were assessed 24 weeks after the completion of the treatment. SVR24 was defined as undetectable serum HCV RNA 24 weeks after the completion of the treatment. All patients were confirmed to have no HCC recurrence using abdominal contrast-enhanced computed tomography (CT) or gadolinium ethoxybenzyl diethylenetriamine pentacetic acid (Gd-EOB-DTPA)-enhanced magnetic resonance imaging (MRI) and ultrasound (US) before the initiation of DAA. Transient elastography (Fibroscan ${ }^{\circ}$ ) with LSM was performed using the $M$ probe at a skin-liver capsule distance of less than $20 \mathrm{~mm}$ and XL probe of more than $20 \mathrm{~mm}$ [19]. Ten validated measurements were performed on each patient and a success rate of least $60 \%$ was considered to be reliable. The LSM of all 119 patients was successfully performed by the $\mathrm{M}$ probe for 109 patients and the XL probe for 10.

\subsection{Follow-Up of Patients}

HCC was diagnosed based on the pattern of nodules on contrast-enhanced CT and Gd-EOB-DTPA-enhanced MR. CT or MR was performed every 6 months 
Table 1. Baseline characteristics of this study with SVR $(n=126)$.

\begin{tabular}{|c|c|c|c|c|c|}
\hline Characteristics & $\begin{array}{c}\text { All } \\
(119)\end{array}$ & $\begin{array}{l}\text { without HCC } \\
\text { treatment before } \\
\text { DAA (105) a }\end{array}$ & $\begin{array}{l}\text { with HCC treatment } \\
\text { before DAA (14) b }\end{array}$ & $\begin{array}{c}\mathbf{P} \\
\mathrm{a} v \mathrm{vs}\end{array}$ & $\begin{array}{l}\text { Recurrence } \\
\quad(8)\end{array}$ \\
\hline Age $(\text { year })^{*}$ & $68(18-91)$ & $67(18-91)$ & $71(56-84)$ & 0.035 & $68(65-81)$ \\
\hline Male:Female & $55: 64$ & 44:61 & $11: 3$ & 0.01 & $5: 3$ \\
\hline $\mathrm{BMI}\left(\mathrm{Kg} / \mathrm{m}^{2}\right)$ & $22.2(14.4-34.9)$ & $22.0(14.4-33.2)$ & $22.5(18.6-34.9)$ & NS & $24.5(20.3-26.8)$ \\
\hline DCV/ASV:SOF/RBV:SOF/LDV:OBV/PTV/rit & $43: 34: 39: 3$ & $35: 31: 36: 3$ & 8:3:3:0 & NS & $3: 2: 3: 0$ \\
\hline $\begin{array}{c}\text { Duration of the follow-up (months)* } \\
\text { DCV/ASV:SOF/RBV:SOF/LD:OBV/PTV/rit } \\
\text { (Median) }\end{array}$ & $\begin{array}{l}32(24-45) \\
41: 31: 30: 26\end{array}$ & $\begin{array}{l}31(24-45) \\
41: 32: 30: 26\end{array}$ & $\begin{array}{c}38(24-42) \\
42: 24: 26:-\end{array}$ & NS & $\begin{array}{c}33(24-45) \\
40: 28: 26:-\end{array}$ \\
\hline $\begin{array}{l}\text { Previous IFN treatment, } \\
\text { Non-responder:Relapse }\end{array}$ & $15: 38$ & $15: 6$ & $0: 2$ & NS & $0: 1$ \\
\hline History of HCC treatments (Resection:RFA) & $14(5: 9)$ & 0 & $14(5: 9)$ & NS & $8(4: 4)$ \\
\hline $\mathrm{DM}$ & 8 & 5 & 3 & NS & 2 \\
\hline Daily alcohol intake $\geq 40 \mathrm{~g} /$ day & 2 & 1 & 1 & NS & 0 \\
\hline HCV RNA (Log IU/ml)* & $5.9(4.5-7.4)$ & $6.1(2.3-7.4)$ & $5.6(4.5-7.3)$ & NS & $6.4(5.1-6.5)$ \\
\hline White cell count $(/ \mu \mathrm{l})^{*}$ & $5000(360-18,000)$ & $5000(360-10,600)$ & $4400(2600-18,000)$ & NS & $6300(3600-9500)$ \\
\hline Hemoglobin $(\mathrm{g} / \mathrm{dl})^{\star}$ & $13.6(7.3-17.2)$ & $13.6(7.3-17.2)$ & $13.2(11.0-14.3)$ & NS & $13.7(12.4-14.3)$ \\
\hline Platelets $\left(10^{4} / \mu \mathrm{l}\right)^{\star}$ Before & $16.5(6.9-80.9)$ & $16.6(6.9-34.8)$ & $12.0(8.0-80.9)$ & NS & $15.5(9.9-28.4)$ \\
\hline SVR24 & $17.8(7.9-62.9)$ & $17.0(6.3-31.2)$ & $11.8(7.1-66.2)$ & NS & $15.0(9.1-34.1)$ \\
\hline Aspartate aminotransferase $(\mathrm{IU} / \mathrm{L})^{*}$ & $39(14-361)$ & $37(14-361)$ & $46(27-212)$ & NS & $69(38-116)$ \\
\hline Alanine aminotransferase $(\mathrm{IU} / \mathrm{L})^{*}$ & $42(12-472)$ & $39(12-472)$ & $51(21-212)$ & NS & $67(23-146)$ \\
\hline Total Bilirubin $(\mathrm{mg} / \mathrm{dl})^{*}$ & $0.72(0.34-5.00)$ & $0.72(0.34-5.00)$ & $0.67(0.5-1.89)$ & NS & $0.80(0.55-1.09)$ \\
\hline $\operatorname{Albumin}(\mathrm{g} / \mathrm{dl})^{*}$ & $4.2(2.6-5.2)$ & $4.2(3.1-5.2)$ & $3.8(2.6-4.6)$ & NS & $4.0(3.1-4.3)$ \\
\hline Creatinine $(\mathrm{mg} / \mathrm{dl})^{*}$ & $0.71(0.46-8.69)$ & $0.81(3.1-8.69)$ & $0.82(0.56-1.15)$ & NS & $0.91(0.67-1.01)$ \\
\hline Alpha-fetoprotein $(\mathrm{AFP})(\mathrm{ng} / \mathrm{ml})^{\star}$ before & $4.0(1-560)$ & $4.0(1-450)$ & $9.0(2-560)$ & 0.01 & $33(4-109)$ \\
\hline SVR24 & $4.0(1-19)$ & $3.0(1.0-19)$ & $17.0(10-47)$ & 0.002 & $8.0(6-133)$ \\
\hline Fib $-4^{*}$ at the start of DAA & $2.90(1.05-5.40))$ & $2.47(1.05-5.11)$ & $3.81(1.52-5.40)$ & NS & $4.35(2.74-5.20)$ \\
\hline Liver stiffness used the probe $\mathrm{M}: \mathrm{XL}$ & 109:10 & $96: 9$ & 13:1 & NS & $7: 1$ \\
\hline LSM0 $(\mathrm{kPa})^{*}$ & $8.7(3.0-28.8)$ & $7.5(3.0-28.8)$ & $15.4(9.8-27.0)$ & $<0.01$ & $18.8(12.1-26.0)$ \\
\hline LSM24 $(\mathrm{kPa})^{*}$ & $6.1(2.5-31.6)$ & $5.8(2.5-31.6)$ & $14.8(5.6-26.0)$ & $<0.01$ & $21.8(9.9-26.6)$ \\
\hline
\end{tabular}

${ }^{*}$ Median (minimum-maximum), NS: not significant, LSM0: LSM at the initiation of DAA, LSM24: LSM 24 months after DAA, DCV/ASV: daclatasvir plus asunaprevir, SOF/RBV: sofosbuvir plus ribavirin, SOF/LDV: sofosbuvir plus ledipasvir, OBV/PTV/rit: ombitasvir plus paritaprevir with ritonavir, RFA: radiofrequency ablation.

before DAA and during the follow-up. LSM was performed at the initiation of DAA (LSM0), at 24 weeks after the start of DAA (LSM24), at 48 weeks after that (LSM48) and at 2 years after that (LSM2y).

\subsection{Statistical Analysis}

Quantitative variables were shown as medians (minimum-maximum). Laboratory data were compared using the $t$-test (parametric data) and chi-squared test. 


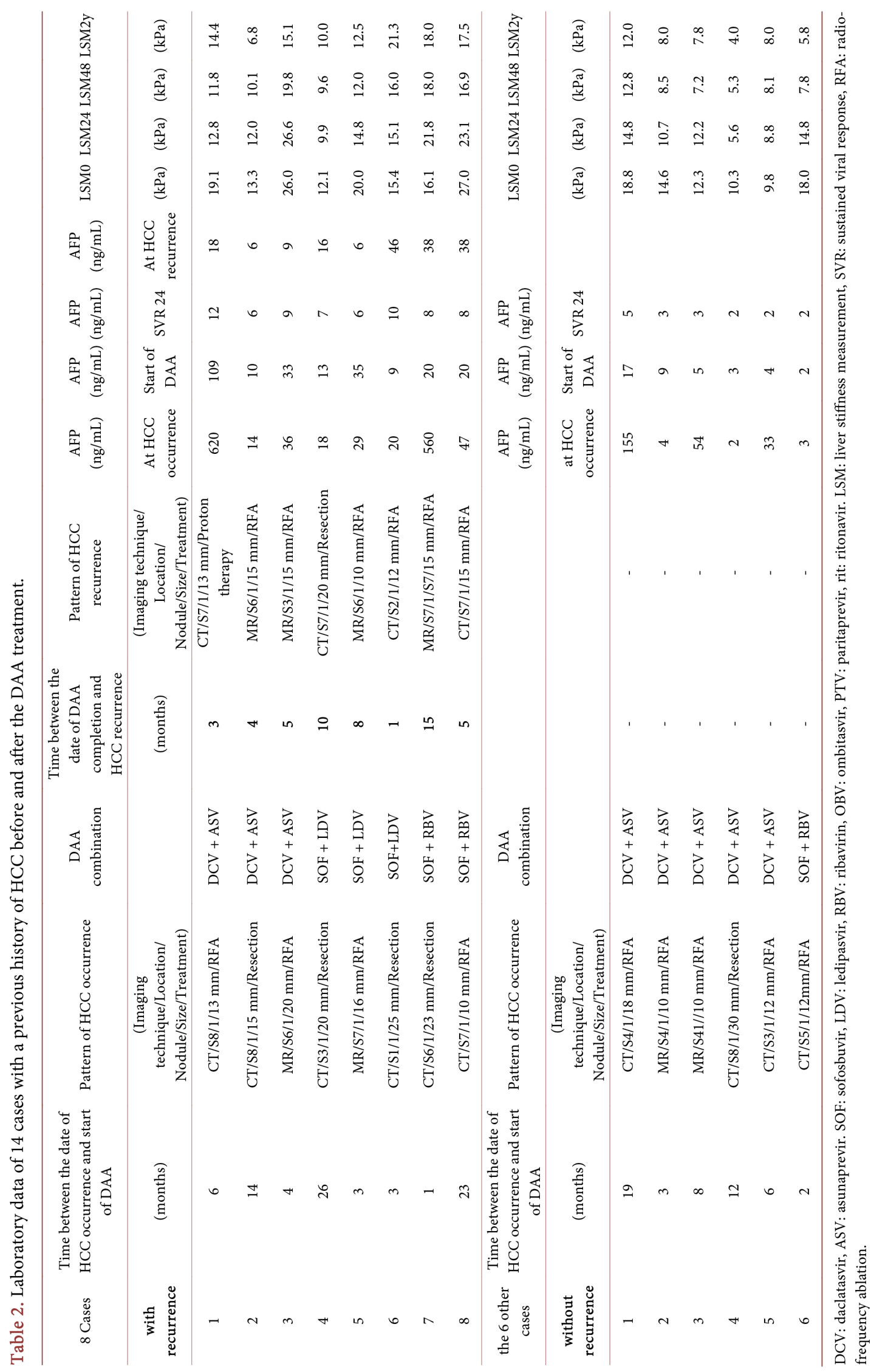


$\mathrm{P}<0.05$ was defined as significant. The HCC development rate was calculated using Kaplan-Meier technique. LSM0, LSM24, and LSM48 were compared using the Mann-Whitney $U$ test and receiver operating characteristic (ROC) curve analyses was performed to obtain the best possible model for the prediction of HCC recurrence. Statistical analyses were performed using the Statistical Package for the Social Science software v. 22 (SPSS, Chicago, IL).

\section{Results}

\subsection{Patient Characteristics}

Table 1 shows patient characteristics. Fourteen out of 119 patients (12\%) had a history of being treated for HCC by RFA or resection and achieved complete responses after previous HCC treatments before the initiation of DAA. The median age of patients was 68 years, and there were 55 males and 64 females. The median follow-up period was 32 months: DCV plus ASV 41 months, SOF plus RBV 31 months, SOF plus LDV 30 months and OBV plus PTV with rit 26 months. Median alpha-fetoprotein (AFP) levels before DAA and at SVR24 were both $4.0 \mathrm{ng} / \mathrm{ml}$. Fib-4 before DAA was 2.90 . Median LSM values at the initiation of DAA (LSM0) and at 24 months after the treatment (LSM24) were $8.7(3.0$ - 28.8) $\mathrm{kPa}$ and $6.1(2.5$ - 31.6), respectively.

Univariate analyses demonstrated significant different for baseline characteristics of age, gender, AFP and LSM between the patients with and without HCC treatments before DAA ( $\mathrm{P}=0.035,0.01,<0.01$, and $<0.01$, respectively).

\subsection{Development of HCC}

Three out of 105 patients without a HCC treatment history (2.9\%) subsequently developed HCC seven, nine, and 25 months after the DAA treatment. Eight out of fourteen patients (57\%) with a history of HCC treatments subsequently showed radiological HCC recurrence a few months (one-fifteen months) after the DAA treatment. Figure 1 shows the cumulative HCC incidence after DAA. The 1-, 2- and 3-year HCC development rates were 1.9\%, 1.9\% and 3.5\% for recurrence, and $43 \%, 51 \%$ and $57 \%$ for recurrence, respectively. Table 2 shows the laboratory data of 14 cases with a previous history of HCC before and after the DAA treatment. Only one HCC nodule was present in each patient at the previous occurrence of HCC and was diagnosed by CT or MR. AFP decreased to less than $10 \mathrm{ng} / \mathrm{ml}$ in six out of eight recurrent patients, while two patients had AFP higher than $10 \mathrm{ng} / \mathrm{ml}$ at SVR24, and five had higher than $10 \mathrm{ng} / \mathrm{ml}$ at the HCC diagnosis. A complete response to the treatment of HCC by ablation or resection was obtained. In patients with HCC recurrence LSM0 increased to more than $12.0 \mathrm{kPa}$ (12.1 - 27.0), LSM24 increased from 9.9 to $26.6 \mathrm{kPa}$ and LSM48 from 9.6 to $19.8 \mathrm{kPa}$. The five other patients with LSM values less than $12 \mathrm{kPa}$ at the last Fibroscan showed no recurrence (Table 2).

\subsection{Variations in LSM during the Follow-Up}

Figure 2 shows the courses of LSM in all patients. 


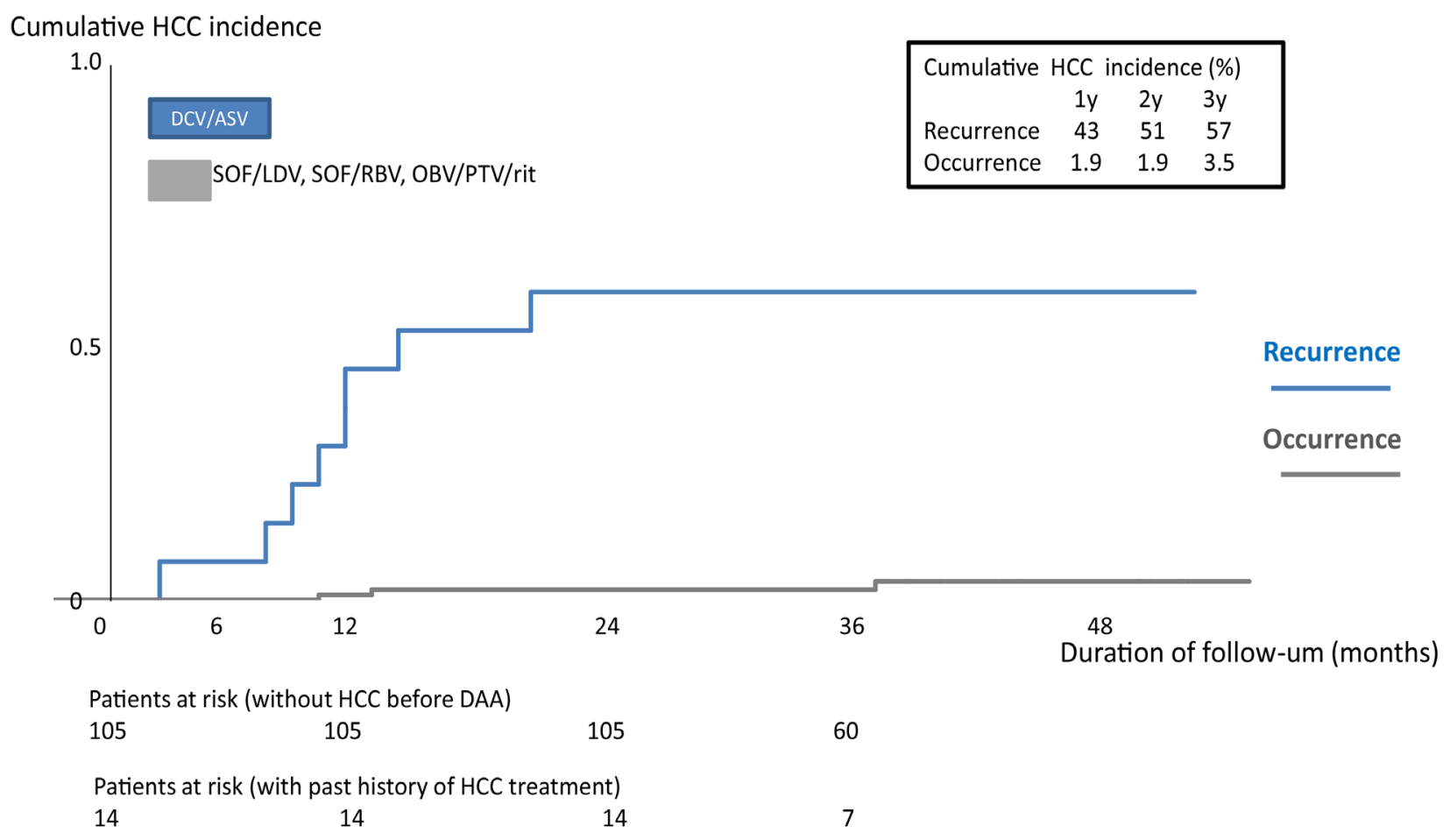

Figure 1. Cumulative HCC incidence.

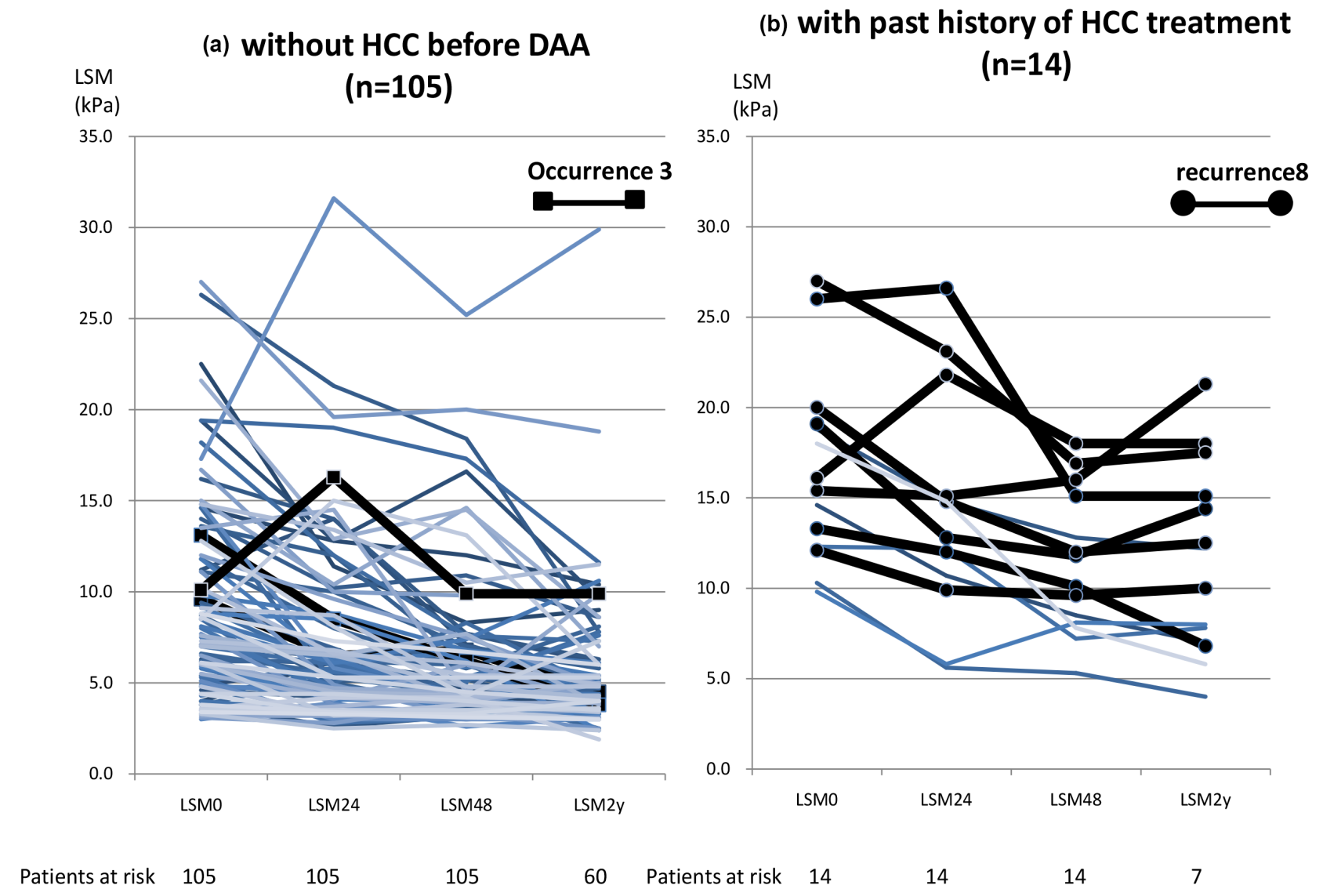

Figure 2. Courses of LSM. 
One hundred and five patients without HCC before DAA who underwent LSM are shown in Figure 2(a). Their LSM0, LSM24, LSM48 and LSM2y were 7.5 (3.0 - 27.0), 6.0 (2.5 - 31.6), 4.6 (2.6 - 25.2) and 4.4 (3.1 - 29.9) kPa, respectively, showing significant improvements. Three out of 105 patients (2.9\%) subsequently developed HCC and their LSM0 were 9.6, 13.1, 10.1, LSM24 6.5, 8.5, 16.3, LSM48 6.8, 6.2, 9.9 and LSM2y 4.5, 3.8, $9.9 \mathrm{kPa}$, respectively (closed squares). Fourteen patients who underwent LSM with a previous history of HCC treatments are shown in Figure 2(b). Their LSM values also significantly improved: LSM0, LSM24, LSM48 and LSM2y were 16.1 (9.8 - 27.0), 14.8 (5.6 26.6), $11.8(5.3$ - 18.0) and $12.2(4.0-21.3) \mathrm{kPa}$, respectively. LSM0 in the eight patients with recurrent HCC increased from 12.1 to $27.0 \mathrm{kPa}$ and LSM24 increased from 9.9 to $26.6 \mathrm{kPa}$ (closed circles). On the other hand, the six other patients without recurrence had LSM values that were less than $12.0 \mathrm{kPa}$ at last (Figure 2(b)).

\subsection{Relationship between LSM and the Recurrence of HCC after SVR}

Since the univariate analysis identified a relationship between LSM values and the recurrence of HCC after SVR, we assessed the cut-off values of these factors for predicting HCC recurrence using an ROC analysis. Based on the ROC analysis, LSM0 15.4, LSM24 12.8 and LSM48 $9.6 \mathrm{kPa}$ were identified as cut-off values, respectively. AUC were $0.77,0.79$ and 0.92 respectively. Therefore, LSM after the DAA treatment was more useful than that before for predicting HCC recurrence (Figure 3(a)). The ROC analysis of AFP was not reliable because AFP data were not normally distributed (Figure 3(b)).

\section{Discussion}

DAA is used in the treatment of hepatitis $\mathrm{C}$ worldwide because of its safety and high efficacy, including patients with advanced fibrosis and a history of HCC treatments. DAA has recently been reported to increase the risk of HCC in HCV-infected patients, particularly in those received DAA after the treatment of HCC. HCC recurrence rates after DAA therapy with a previous history of HCC treatments vary in different studies due to the heterogeneity of study settings. In the present study, among fourteen patients infected with HCV who achieved SVR by DAA after complete responses to the treatment of HCC by RFA or resection, eight (57\%) subsequently developed radiological HCC recurrence after a few months (one-fifteen months). Reig M reported that HCC recurrence was recognized in $27.6 \%$ patients within 5.7 months of the initiation of DAA (10). In that study, some patients were treated with transcatheter arterial chemoembolization (TACE), which is potentially non-curative therapy. All our patients were treated with potentially curative treatments, resection and RFA, HCC recurrence was noted between one and fifteen months after three kinds of DAA; DCV plus ASV three cases, SOF plus LDV three cases and SOF plus RBV two cases. We reported that three out of eight patients treated with resection and RFA 
(a). LSM

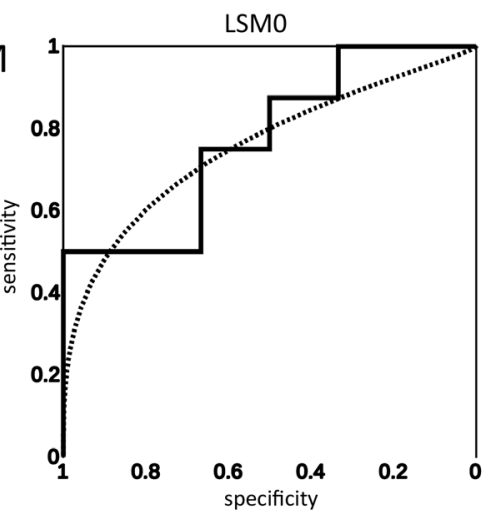

(b). AFP
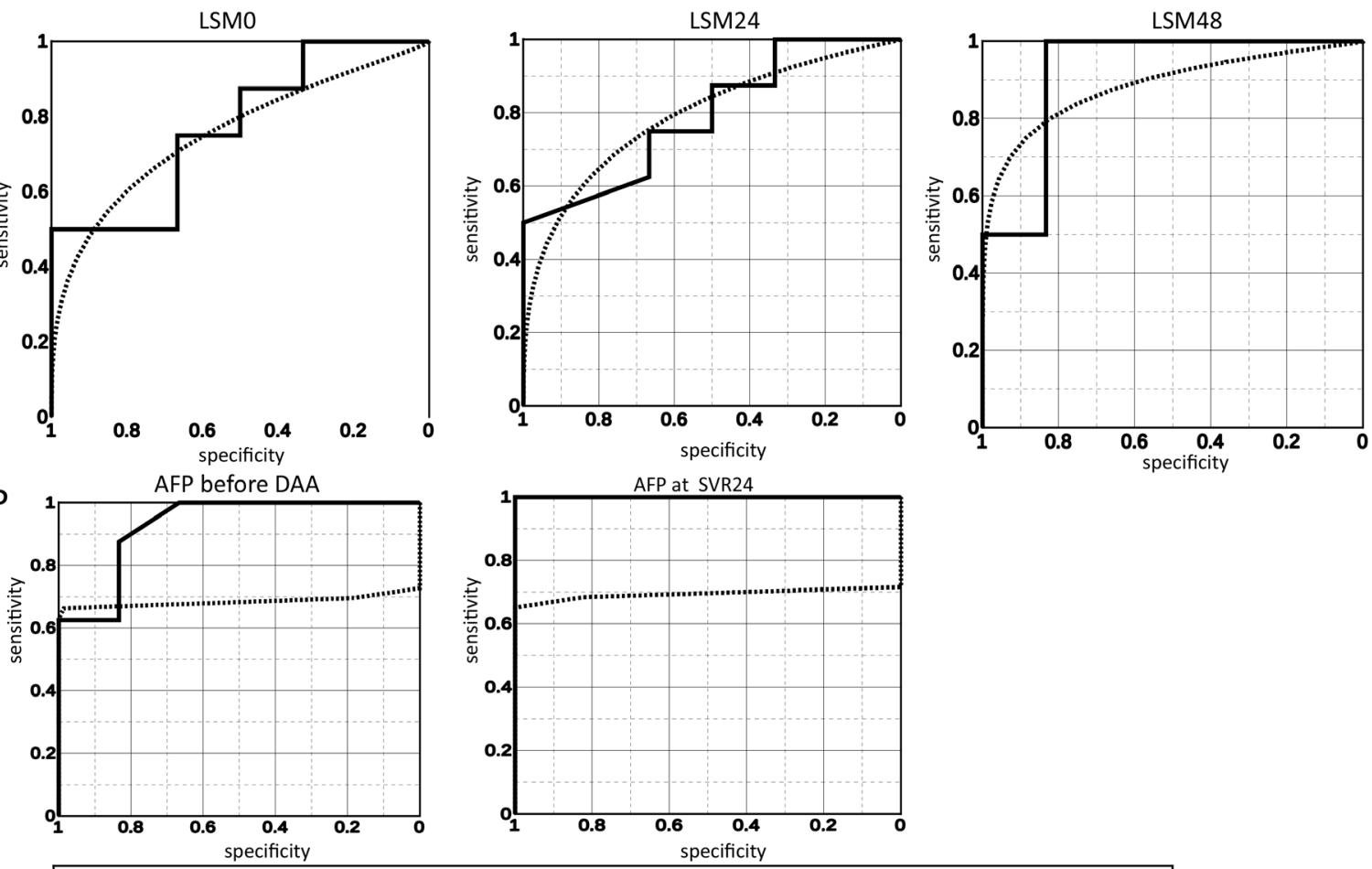

\begin{tabular}{|c|c|c|c|c|c|c|}
\hline Variables & AUC & $95 \% \mathrm{Cl}$ & Cut-off & Sensitivity & Specificity & accuracy \\
\hline LSMO & 0.77 & $0.52-1$ & 15.4 & 0.75 & 0.67 & 0.71 \\
\hline LSM24 & 0.79 & $0.55-1$ & 12.8 & 0.75 & 0.67 & 0.71 \\
\hline LSM48 & 0.92 & $0.55-1$ & 9.6 & 1 & 0.83 & 0.93 \\
\hline AFP before DAA & 0.93 & $0.79-1$ & 10 & 0.85 & 0.83 & 0.86 \\
\hline AFP at SVR24 & 1.00 & $1-1$ & 6.0 & 1.00 & 1.00 & 1.00 \\
\hline
\end{tabular}

Figure 3. ROC curve for prediction of HCC.

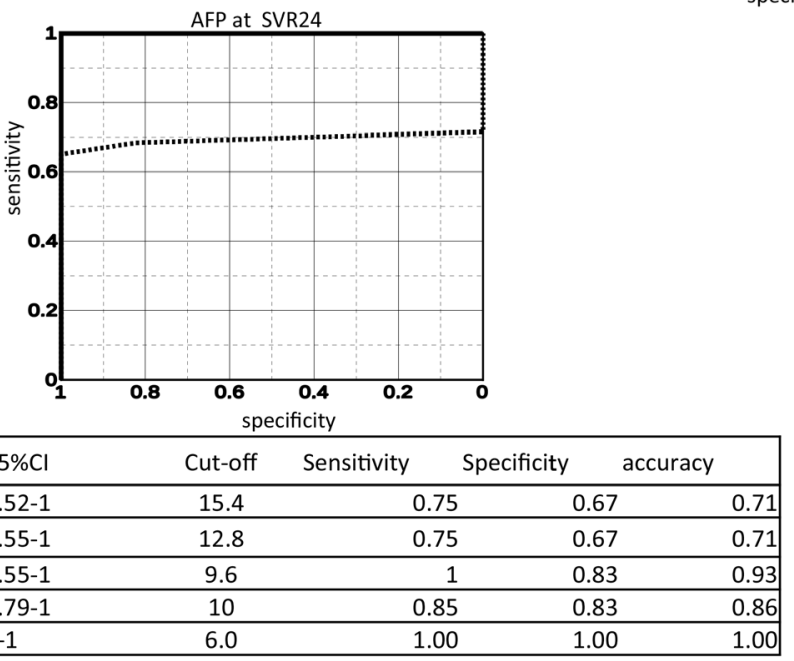

developed HCC recurrence from three to five months after DCV plus ASV (16). DCV plus ASV was approved only in Japan and Korea of over the world, therefore the aim of the present study was to prospectively evaluate the relationship between the recurrence of HCC and liver stiffness values after the successful treatment of patients with HCV infection with all kinds of DAA.

LSM0 in fourteen patients with a previous history of HCC treatments was 15.4 (9.8 - 27.0) $\mathrm{kPa}$. A LSM value of more than $12.0 \mathrm{kPa}$ was previously reported to indicate severe fibrosis [20], and twelve out of fourteen patients with values higher than $12.0 \mathrm{kPa}(12.1$ - 27.0) showed the development of liver cirrhosis. Conti F, et al. reported that $\mathrm{HCC}$ recurrence more significantly recognized in the patients with LSM values over $21.5 \mathrm{kPa}$ before DAA therapy [15]. Though LSM values over $21.5 \mathrm{kPa}$ is recognized to be liver cirrhosis, in the present study only Cases 3 and 8 were over $21.5 \mathrm{kPa}$ (Table 2). Cheung M C M, et al. reported that the patients with decompensated cirrhosis after DAA therapy improved liver function but not decreased the rates of HCC occurrence [21]. The examination of the relationship between the HCC recurrence and the changes in LSM values after SVR with DAA has not been reported till now. We prospectively followed-up or over two years and analyzed changes in LSM in HCV-infected patients with and without a previous history of HCC. LSM values at the initiation 
of DAA (LSM0) in the eight patients with recurrent HCC increased from 12.1 to $27.0 \mathrm{kPa}$, showing the development of liver cirrhosis. Moreover, LSM24 seven out of eight these patients increased from 12.0 to $26.6 \mathrm{kPa}$. On the other hand, the LSM values of the six other cases without recurrence gradually decreased to less than $12.0 \mathrm{kPa}$ after DAA. Based on the ROC analysis, LSM0 15.4, LSM24 12.8 and LSM48 $9.6 \mathrm{kPa}$ were identified as cut-off values. Regarding LSM after the DAA treatment, LSM24 and LSM48 were more useful than LSM0 for predicting HCC recurrence (Figure 3(a)). A point of no return for cirrhosis may exist and be identified using LSM values. In HCV patients previously treated for HCC with high LSM values not only before but also after DAA, the risk of HCC recurrence may still be high.

The patients obtained SVR by IFN treatments showed the significant regression of LSM [12]. The LSM values in the patients obtained SVR by DAA also were significantly reduced during follow-ups [13] [14]. This early LSM improvement was recognized to be attributed by a decrease in the inflammation of liver tissue. Even in the six other cases without recurrence LSM values showed significant decreases during the follow-up (Table 2). Because the elevation of LSM affected by the artifacts due to liver inflammation were no longer observed in LSM24 and LSM48, the decreases of LSM24 and LSM48 are considered to reflect a true improvement of liver fibrosis. LSM0, LSM24, LSM48 and LSM2y of the patients without HCC before DAA at showed significant improvements (Figure 3(a)). However, LSM24 in recurrent patients increased from 9.9 to 26.6 $\mathrm{kPa}$ which was high, and the regression of fibrosis was not recognized before HCC recurrence. In patients previously treated for HCC with high LSM values before and after DAA, the risk of HCC recurrence may still be high.

Post-AFP levels after IFN therapy were previously reported to correlate with the occurrence of HCC [22]. In the present study, the ROC analysis of AFP was not reliable because AFP data were not normally distributed. A large prospective study involving LSM and AFP needs to be conducted. There are limitations to this study. First, the sample size is small in single center study. Second, the patients with a history of HCC treatment were only fourteen, because we restricted their therapy curative (RFA and resection).

Kobayashi reported that SVR by DAA reduces HCC incidence of HCC, the effects of DAA on HCC occurrence remain unknown because of a lack of prospective evidence [23]. In the present study, three out of 105 patients without a HCC treatment history subsequently developed HCC seven, nine, and 25 months after the DAA treatment. The relationship between LSM and the occurrence of HCC currently remains unknown. A large prospective study with properly selected populations and a long-term follow-up needs to be conducted.

\section{Conclusion}

In conclusion, HCV patients previously treated for HCC with high LSM values before and after DAA are still at an elevated risk of HCC recurrence, particularly those with LSM24 higher than $12.8 \mathrm{kPa}$ and LSM48 higher than $9.6 \mathrm{kPa}$. These 
patients need to be closely monitored while LSM values are high, even after the achievement of SVR.

\section{Ethical Approval}

The present study was approved by the Ethics Committee of National Hospital Organization Nagoya Medical Center, in accordance with the Helsinki Declaration.

\section{Informed Consent}

Informed consent was obtained from all individual participants included in the study.

\section{Consent for Publication}

I agree Consent for publication.

\section{Availability of Data and Material}

Our data are available at National Hospital Organization Nagoya Medical Center.

\section{Competing Interests}

Conflicts of Interest: Yasuhito Tanaka received lecture fees from Bristol-Myers Squibb Company, MSD K.K., Chugai Pharmaceutical Co., Ltd., Janssen Pharmaceutical K.K., and Gilead Sciences. Yasuhito Tanaka received Commercial research funding from Bristol-Myers Squibb Company, Chugai Pharmaceutical Co., Ltd., and AbbVie Inc. Noboru Hirashima, Hiroaki Iwase, Masaaki Shimada, Noboru Urata, and Etsuko Iio have no conflicts of interest.

\section{Funding}

We have no funding.

\section{Authors' Contributions}

All the authors contributed the concept, writing and checking of this manuscript.

\section{Acknowledgements}

The authors would like to thank Norio Sugimoto for excellent advice on data analysis.

\section{References}

[1] Lawitz, E., Sulkowski, M.S., Ghalib, R., Rodriguez-Torres, M., Younossi, Z.M., Corregidor, A., DeJesus, E., et al. (2014) Simeprevir plus Sofosbuvir, with or without Ribavirin, to Treat Chronic Infection with Hepatitis C Virus Genotype 1 in Non-Responders to Pegylated Interferon and Ribavirin and Treatment-Naive Pa- 
tients: The COSMOS Randomized Study. The Lancet, 384, 1756-1765.

https://doi.org/10.1016/S0140-6736(14)61036-9

[2] Poordad, F., Hezode, C., Trinh, R., Kowdley, K.V., Zeuzem, S., Agarwal, K., Shiffman, M.L., et al. (2014) ABT-450/R-Ombitasvir and Dasabuvir with Ribavirin for Hepatitis C with Cirrhosis. The New England Journal of Medicine, 370, 1973-1982. https://doi.org/10.1056/NEJMoa1402869

[3] Lawitz, E., Gane, E., Pearlman, B., Tam, E., Ghesquiere, W., Guyader, D., Alric, L., et al. (2015) Efficacy and Safety of 12 Weeks Versus 18 Weeks of Treatment with Grazoprevir (MK-5172) and Elbasvir (MK-8742) with or without Ribavirin for Hepatitis C Virus Genotype 1 Infection in Previously Untreated Patients with Cirrhosis and Patients with Previous Null Response with or without Cirrhosis (C-WORTHY): A Randomized, Open-Label Phase 2 Trial. The Lancet, 385, 1075-1086.

https://doi.org/10.1016/S0140-6736(14)61795-5

[4] Afdhal, N., Reddy, K.R., Nelson, D.R., Lawitz, E., Gordon, S.C., Schiff, E., Nahass, R., et al. (2014) Ledipasvir and Sofosbuvir for Previously Treated HCV Genotype 1 Infection. The New England Journal of Medicine, 370, 1483-1493. https://doi.org/10.1056/NEJMoa1316366

[5] Kumada, H., Suzuki, Y., Ikeda, K., Toyota, J., Karino, Y., Chayama, K., Kawakami, Y., et al. (2014) Daclatasvir plus Asunaprevir for Chronic HCV Genotype 1B Infection. Hepatology, 59, 2083-2091. https://doi.org/10.1002/hep.27113

[6] Manns, M., Pol, S., Jacobson, I.M., Marcellin, P., Gordon, S.C., Peng, C.Y., Chang, T.T., et al. (2014) All-Oral Daclatasvir plus Asunaprevir for Hepatitis C Virus Genotype 1B: A Multinational, Phase 3, Multicohort Study. The Lancet, 384, 1597-1605. https://doi.org/10.1016/S0140-6736(14)61059-X

[7] Chayama, K., Notsumata, K., Kurosaki, M., et al. (2015) Randomized Trial of Interferon- and Ribavirin-Free Ombitasvir/Paritaprevir/Ritonavir in Treatment-Experienced Hepatitis C Virus-Infected Patients. Hepatology, 61, 1523-1532. https://doi.org/10.1002/hep.27705

[8] Zeuzem, S., Dusheiko, G.M., Salupere, R., et al. (2014) Sofosbuvir and Ribavirin in HCV Genotype 2 and 3. The New England Journal of Medicine, 370, 1993-2001. https://doi.org/10.1056/NEJMoa1316145

[9] Iio, E., Shimada, N., Abe, H., et al. (2017) Efficacy of Daclatasvir/Asunaprevir According to Resistance-Associated Variants in Chronic Hepatitis C with Genotype 1. Journal of Gastroenterology, 52, 94-103. https://doi.org/10.1007/s00535-016-1225-x

[10] Reig, M., Marino, Z., Perello, C., et al. (2016) Unexpected High Rate of Early Tumor Recurrence in pAtients with HCV-Related HCC Undergoing Interferon-Free Therapy. Journal of Hepatology, 65, 719-726.

https://doi.org/10.1016/j.jhep.2016.04.008

[11] Morgan, R.L., Baack, B., Smith, B.D., et al. (2013) Eradication of Hepatitis C Virus Infection and the Development of Hepatocellular Carcinoma: A Meta-Analysis of Observational Studies. Annals of Internal Medicine, 158, 329-337. https://doi.org/10.7326/0003-4819-158-5-201303050-00005

[12] Masias, J., Rivero, A., Cifucentes, C., et al. (2013) Sustained Viral Response to Pegylated Interferon plus Ribavirin Leads to Normalization of Liver Stiffness in Hepatitis C Virus-Infected Patients. Enfermedades Infecciosas y Microbiología Clínica, 31, 424-429. https://doi.org/10.1016/j.eimc.2012.12.004

[13] Bachofner, J.A., Valli, P.V., Kroger, A., et al. (2017) Direct Antiviral Agent Treatment of Chronic Hepatitis C Results in Rapid Regression of Transient Elastography and Fibrosis Markers Fibrosis-4 Score and Aspartate Aminotransferase-Platelet Ra- 
tio index. Liver International, 37, 369-376. https://doi.org/10.1111/liv.13256

[14] Martini, S., Sacco, M., Strona, S., et al. (2017) Impact of Viral Eradication with Sofosbuvir-Based Therapy on the Outcome of Post-Transplant Hepatitis C with Severe Fibrosis. Liver International, 37, 62-70. https://doi.org/10.1111/liv.13193

[15] Conti, F., Buonfiglioli, F., Scuteri, A., et al. (2016) Early Occurrence and Recurrence of Hepatocellular Carcinoma in HCV-Related Cirrhosis Treated with Direct-Acting Antivirals. Journal of Hepatology, 65, 727-733. https://doi.org/10.1016/j.jhep.2016.06.015

[16] Hirashima, N., Iwase, H., Shimada, M., Ryuge, N., Ureta, N., et al. (2016) Early Recurrence of Hepatocellular Carcinoma in Patients with HCV Infection Followed by Transient Elastography for Two Years after Successful Treatment with Daclatasvir plus Asunaprevir. Gastroenterology, Hepatology and Endoscopy, 2, 1-5.

[17] Karino, Y., Toyota, J., Ikeda, K., Suzuki, F., Chayama, K., Kawakami, Y., Ishikawa, H., et al. (2013) Characterization of Virologic Escape in Hepatitis C Virus Genotype 1B Patients Treated with the Direct-Acting Antivirals Daclatasvir and Asunaprevir. Journal of Hepatology, 58, 646-654. https://doi.org/10.1016/j.jhep.2012.11.012

[18] McPhee, F., Suzuki, Y., Toyota, J., Karino, Y., Chayama, K., Kawakami, Y., Yu, M.L., et al. (2015) High Sustained Virologic Response to Daclatasvir plus Asunaprevir in Elderly and Cirrhotic Patients with Hepatitis C Virus Genotype 1B Without Baseline NS5A Polymorphisms. Advances in Therapy, 32, 637-649. https://doi.org/10.1007/s12325-015-0221-5

[19] Durango, E., Dietrich, C., Seitz, H.K., et al. (2013) Direct Comparison of the FibroScan XL and M Probes for Assessment of Liver Fibrosis in Obese and Nonobese Patients. Hepatic Medicine, 5, 43-52.

[20] Castera, L., Forns, X. and Alberti, A. (2008) Non-Invasive Evaluation of Liver Fibrosis Using Transient Elastography. Journal of Hepatology, 48, 835-847. https://doi.org/10.1016/j.jhep.2008.02.008

[21] Cheung, M.C.M., Walker, A.J., Hudson, B.E., et al. (2016) Outcome after Successful Direct-Acting Antiviral Therapy for Patients with Chronic Hepatitis C and Decompensated Cirrhosis. Journal of Hepatology, 65, 741-747. https://doi.org/10.1016/j.jhep.2016.06.019

[22] Asahina, Y., Tsuchia, K., Nishimura, T., et al. (2013) $\alpha$-Fetoprotein Levels after Interferon Therapy and Risk of Hepatocarcinogenesis in Chronic Hepatitis C. Hepatology, 58, 1253-1262. https://doi.org/10.1002/hep.26442

[23] Kobayashi, M., Suzuki, F., Fujiyama, S., Kawamura, Y., Sezaki, H., et al. (2017) Sustained Virological Response by Direct Antiviral Agents Reduces the Incidence of Hepatocellular Carcinoma in Patients with HCV Infection. Journal of Medical Virology, 89, 476-483. 


\section{Abbreviation}

DAA: Direct-acting antiviral agents

DCV: Daclatasvir

ASV: Asunaprevir

$\mathrm{HCV}$ : Hepatitis $\mathrm{C}$ virus

IFN: Interferon

SOF: Sofosbuvir

LDV: Ledipasvir

OBV: Ombitasvir

PTV: Paritaprevir

Rit: Ritonavir

RBV: Ribavirin

SVR: Sustained virological responses

NS5A: Non-structural protein $5 \mathrm{~A}$

LSM: Liver stiffness measurements

TACE: Transcatheter arterial chemoembolization

HCC: Hepatocellular carcinoma

CT: Computed tomography

Gd-EOB-DTPA: Gadolinium ethoxybenzyl diethylenetriamine pentaacetic acid MRI: Magnetic resonance imaging 